\title{
Practical Method to Estimate the Standard Deviation in Absorption Coefficients Measured with THz Time-Domain Spectroscopy
}

\author{
Saroj R. Tripathi \\ Shizuoka University, 3-5-1 Johoku, Hamamatsu, Shizuoka 432-8011, Japan \\ Makoto Aoki \\ Shizuoka University, 3-5-1 Johoku, Hamamatsu, Shizuoka 432-8011, Japan \\ Kento Mochizuki \\ Shizuoka University, 3-5-1 Johoku, Hamamatsu, Shizuoka 432-8011, Japan
}

Iwao Hosako

National Institute of Information and Communication Technology, 4-2-1 Nukui-Kitamachi, Tokyo 184-8795, Japan

Toshiaki Asahi

Nippon Mining and Metals Co. Ltd, 3-17-35, Niizo-Minami, Toda, Saitama 335-8502, Japan

Norihisa Hiromoto

Shizuoka University, 3-5-1 Johoku, Hamamatsu, Shizuoka 432-8011, Japan

\section{Corresponding Author}

Norihisa Hiromoto

Shizuoka University, 3-5-1 Johoku, Hamamatsu, Shizuoka 432-8011, Japan

Tel/Fax: +81-53-478-1351

Email: dnhirom@ipc.shizuoka.ac.jp

\begin{abstract}
:
A model of standard deviation in the intensity spectrum of electric field observed with the terahertz time-domain spectroscopy (THz-TDS) is proposed to estimate the random error in the transmittance and absorption coefficients from a single or a few measurements of a sample. The proposed standard deviation which is derived on the basis of the statistical standard deviation and noise floor of intensity spectrum of reference fits well to the standard deviation of transmittance as well as absorption coefficient computed statistically. This study contributes the simple and computationally efficient method to demonstrate the accuracy in optical constants like imaginary part of refractive index and absorption coefficients measured using the THz-TDS.
\end{abstract}




\section{Keywords:}

Terahertz wave, THz time-domain spectroscopy, optical constant, random error Classification codes:

87.64.k-; 78.20.ci; 29.85.Fj

\section{Introduction}

Terahertz time-domain spectroscopy (THz-TDS) has been widely used to study the spectroscopic characteristics of the variety of the materials [1-3] like dielectrics [4], semiconductors [4-5], bio-molecules [6], liquids [7], pharmaceutical products [8] and so on in the spectral region spanning from 0.1 to $3 \mathrm{THz}$. Most spectroscopic measurements involve the recording of a time-domain pulse of $\mathrm{THz}$ electric field transmitted through a sample under investigation placed on the path of the terahertz radiation and a reference signal recorded in the absence of the sample. The time-domain waveforms are converted to the frequency-domain by the complex Fourier transform to obtain the intensity and phase spectra. These spectra are processed further to calculate the transmittance and phase differences which are used to derive the frequency dependent dielectric properties like complex refractive index, complex dielectric constant and absorption coefficient.

The measured spectra of sample and reference electric fields using THz-TDS and the dielectric properties (also called as optical constants) obtained using these electric fields are prone to have systematic and random errors and the precision is limited by them [e.g., see ref. 9]. The systematic error is due to error in measure of sample-thickness measurements (including parallelism and roughness of the surfaces), sample misalignment, error in optical delay distance etc. and these errors could be eliminated or reduced to some extent by making effort in sample preparation, spectrometer calibration and so on. However, the random errors which are caused mainly by laser power fluctuation, electronic noise in devices, optical and mechanical instability can not be removed completely and it becomes necessary to quantify them with statistical analysis by taking number of observations.

Previously, a method to identify the dynamic range from one reference spectrum and the detectable limit of absorption coefficient from the sample spectrum has been reported [10], but this does not provide the information on the random error in measured data. The methodology to determine the standard deviation in intensity spectrum has been reported by taking large number of measurements [11]. As one scan of measurement in THz-TDS requires several to ten minutes, more than several hours are necessary to take enough number of sample and reference data for evaluating the statistical standard deviations in the measured optical parameters with an ordinary frequency resolution, which is usually difficult and not practical to execute always for many samples. One or a few pair of reference-and-sample data are usually expected to obtain the optical parameters of each sample by the THz-TDS, in which case the estimation of errors can not be done by statistical methods. Therefore, in this study we provide the method to estimate the error in the transmittance and absorption coefficient even if a sample is measured 
only one time with THz-TDS by using the statistical standard deviations in the intensity spectra derived from many number of reference data.

\section{Measurements}

We took a number of reference and sample time-domain waveforms with a THz-TDS system to examine the error characteristics in the measured data. The THz-TDS system used for the measurement is the Rayfact Spectera [12] (Tochigi Nikon Corporation) consisting of a compact femto-second fiber laser (Femtolite, IMRA Inc.) with a pulse width of less than 100 fs and 20 $\mathrm{mW}$ average power and LT-GaAs photoconductive antennas as THz emitter and detector. Three ZnTe crystals with different thickness $(0.767 \mathrm{~mm}, 1.496 \mathrm{~mm}$ and $2.976 \mathrm{~mm})$ supplied commercially by Nippon Mining and Metals Co. Ltd. [13] are used as samples and they have very low absorption coefficient in the frequency range of 0.2 to $3 \mathrm{THz}$ [14]. The time-domain data of 1024 points is taken in one scan of time delay line with the frequency resolution of 0.024 $\mathrm{THz}\left(0.814 \mathrm{~cm}^{-1}\right)$, which needs about 10 minutes for each scan. The data is observed in the sequence like $R_{0}, S_{0}, S_{1}, R_{1}, R_{2}, S_{2}, \ldots R_{k}, S_{k}, \ldots, S_{m-1}, S_{m}, R_{m}$, where $R$ and $S$ are the reference and sample data respectively, and $k$ and $m$ of subscripts are integers. The $R_{k}$ and $S_{k}$ with the same subscript means a pair of reference and sample data which are close to each other in time at which they are measured. Twelve, sixteen and sixteen data sets of reference and sample waveforms were recorded for the $0.767 \mathrm{~mm}, 1.496 \mathrm{~mm}$ and $2.976 \mathrm{~mm}$ thick ZnTe crystal respectively.

\section{Derivation and modeling of standard deviation}

The time-domain pulses of electric field suffered from the random error are characterized by the temporal shift of pulse position and pulse shape, which are approximately represented by a position in time and amplitude of the largest pulse in the waveform respectively. The variation in position and amplitude of the peak depend on time of an order of hour, which showed good correlation between reference and sample data. It is possible to correct the variation in position and amplitude of the largest peak pulse by performing a data processing prior to taking Fourier transform. We therefore adjusted the waveforms so that respective peak amplitude become equal to the averaged peak height. After conversion of the waveform data from time-domain to frequency-domain by the Fourier transform, this pre-process have brought a good effect to improve the standard deviation in the intensity spectra. As a result, the standard deviation in the transmittance derived through the error-propagation from reference and sample spectra has become almost equal to the statistical standard deviation which is calculated from the transmittances obtained using each pair of reference and sample spectra. In other words, this means the data taken more closely in time has smaller variances.

The intensity spectra of reference and sample are derived by the procedure explained above for the $\mathrm{ZnTe}$ crystals with three different thicknesses. Figure 1 displays the average of 12 data 
of $0.767 \mathrm{~mm} \mathrm{ZnTe}$ sample and reference spectra along with their respective standard deviations calculated by statistical mathematics. Beyond the detectable upper frequency limit, the intensity spectrum approaches a flat noise level, which is called as the noise floor $[10,15]$. The intensity spectra of reference and sample have the noise floor above $7 \mathrm{THz}$ and $4.5 \mathrm{THz}$ respectively. The remarkable feature in Fig.1 is that the standard deviations of reference and sample are much higher than the level of noise floor in the larger intensity region and they are correlated with the respective intensity; the intensity of the sample is smaller than that of the reference due to attenuation caused by the ZnTe crystal and hence the standard deviation of former is smaller than latter's. Spectra of intensity and standard deviation of the reference as well as sample show that the signal-to-noise ratio (SNR) is rapidly improved with an increase in the intensity from the noise floor to a higher level, but the SNR nearly becomes to be unchanged in the high intensity region.

We therefore assume the simple relation between the standard deviation and the intensity as expressed in Eq.(1), implying that the standard deviation is proportional to the intensity and approaches to the noise floor at the lowest intensity signal region.

$$
\sigma_{I}(\omega)=C(I(\omega)-h)+h
$$

where $\omega$ is angular frequency, $\sigma_{I}(\omega)$ is the standard deviation of the intensity, $I(\omega)$ is the mean intensity, $h$ is the noise floor, and $C$ is assumed as a proportionality constant. We calculated the values of $C$ from the standard deviation and intensity spectra of reference and sample using Eq. (1) to verify its validity. Figure 2 shows the values of $C$ decrease from the higher frequency to lower frequency, meaning that SNR is improved, and increase a little below $1 \mathrm{THz}$, implying the SNR becomes worse though the intensity still increases there. These facts suggest that the SNR is almost unchanged around the high intensity region, and hence the standard deviation becomes explicitly proportional to the intensity and $C$ can be taken as a constant independent from frequency. Eq. (1) provides the simplest computational model using the constant $C$ to derive the standard deviation from the intensity spectrum. The value of $C$ does not depend on samples, since it is common in the reference and sample as shown in Fig. 2. Therefore, it can be determined by the intensity and standard deviation spectrum of only the reference in the high intensity signal region. The origin of intensity-correlated random error in $\mathrm{THz}$ signal is presently unknown, but it is possibly related to the emission or detection process of $\mathrm{THz}$ wave because intensity fluctuation of femto-second fiber laser as well as its variation during the time delay scanning are much smaller than the observed standard deviation in the $\mathrm{THz}$ signal.

If we know the $C$ and the noise floor for a sequence of reference intensity spectrum measured with a THz-TDS, we can estimate the standard deviation of the sample intensity spectrum even if it is taken only one time. We can afford to measure different samples in the data-taking sequence of $R_{0}, S_{0}, S_{1}, R_{1}, R_{2}, S_{2}, \ldots R_{k}, S_{k}, \ldots, S_{m-1}, S_{m}, R_{m}$ though the reference data should be continuously taken under the same condition. 


\section{Estimation of standard deviations in transmittance and absorption coefficients.}

Transmittance was calculated as $T(\omega)=I_{S}(\omega) / I_{R}(\omega)$ from a pair of sample and reference intensity spectra, $I_{S}(\omega)$ and $I_{R}(\omega)$, and the average and standard deviation of the transmittance was calculated by statistical mathematics from the 12 data set of the $0.767 \mathrm{~mm}$ thick ZnTe sample and reference as shown in Fig. 3. Such a standard deviation is called hereafter as the statistical $\sigma$. It can be seen that the statistical $\sigma_{T}$ in transmittance becomes almost equal to the transmittance above around $3.2 \mathrm{THz}$ which is the detectable upper frequency limit of the transmittance.

We calculated the standard deviation in the intensity spectrum of $0.767 \mathrm{~mm}$ thick ZnTe sample and the corresponding reference using Eq. (1) by taking the constant $C$ as 0.0125 . The standard deviation thus calculated is referred hereafter as the estimated $\sigma$. The estimated $\sigma$ 's of the reference and sample are denoted as $\sigma_{R}$ and $\sigma_{S}$, by which $\sigma_{T}$ is also calculated using the error-propagation law [16] as

$$
\sigma_{T}=T(\omega)\left(\frac{\sigma_{R}^{2}}{I_{R}^{2}(\omega)}+\frac{\sigma_{S}^{2}}{I_{S}^{2}(\omega)}\right)^{1 / 2} .
$$

The estimated $\sigma_{T}$ is displayed together with statistical $\sigma_{T}$ in Fig. 3. This figure shows the estimated $\sigma_{T}$ is well fitted to the statistical $\sigma_{T}$ within the factor of 2 between 0.2 and $3 \mathrm{THz}$. The evaluation of such standard deviation made by the simple model is probably sufficient for the measurements of most samples. We believe the proposed model is important and beneficial to the THz-TDS which has difficulty in gathering enough number of reference-and-sample spectra for calculating the statistical standard deviation.

For comparison, in Fig. 3, we show also a standard deviation in transmittance calculated through the error propagation on the assumption that the noise floors of references and sample are equal to the respective standard deviation in all frequencies and such a standard deviation is called as the $\sigma_{T}$ from the noise floor. The $\sigma_{T}$ from noise floor is also coincides with the transmittance above around $3.2 \mathrm{THz}$ but it is far from the statistical $\sigma_{T}$ and the estimated $\sigma_{T}$ in the high intensity signal region, indicating that the true standard deviation cannot be derived from the noise floor.

The complex refractive index $n_{c}(\omega)=n(\omega)+i k(\omega)$, where $n(\omega)$ and $k(\omega)$ are real and imaginary parts respectively, is calculated from the transmittance and the phase difference between sample and reference signal through iteration calculation. Absorption coefficient is derived from the imaginary part of refractive index by the equation below, where $c$ is the speed of light.

$$
\alpha(\omega)=\frac{2 \omega k(\omega)}{c}
$$


The statistical standard deviation in absorption coefficient $\sigma_{\alpha}$ is calculated statistically using a number of the derived absorption coefficients.

The estimated $\sigma_{k}$ of the imaginary part of refractive index can be computed from the estimated $\sigma_{T}$ using the error-propagation law assuming $\sigma_{k}$ is mainly originated from the random error in $T(\omega)$ by Eq. (4).

$$
\sigma_{k}=\frac{c}{2 d \omega} \cdot \frac{\sigma_{T}}{T(\omega)}
$$

where $d$ is a thickness of the sample. Using general law of the error propagation again, the estimated $\sigma_{\alpha}$ in absorption coefficient can be calculated as

$$
\sigma_{\alpha}=\left(\frac{2 \omega}{c}\right) \sigma_{k}
$$

Figures 4(a), 4(b) and 4(c) display the absorption coefficients $\alpha$ in $\mathrm{cm}^{-1}$, the statistical $\sigma_{\alpha}$ and the estimated $\sigma_{\alpha}$ of the $0.767 \mathrm{~mm}, 1.496 \mathrm{~mm}$ and $2.976 \mathrm{~mm}$-thick ZnTe samples respectively. The value of constant $C$ obtained from the reference data taken during the measurement of these samples were $0.0125,0.0139$ and 0.0096 respectively, which are rather close but a little different from each other. This is because the same THz-TDS instrument is used in the measurements, but the condition of the spectrometer and the environment of the measurement are somewhat changed due to the different measurement date. The estimated $\sigma_{\alpha}$ 's calculated by the method proposed here are well fitted to the statistical $\sigma_{\alpha}$ 's for all samples with different thickness, though the detectable frequency limit in the absorption coefficients are depend on the sample thickness as shown by the vertical dashed line in Fig 4.

\section{Conclusions}

This study proposes a method to estimate the standard deviation in the intensity spectrum measured with the THz-TDS, implying a model of the standard deviation which is proportional to the intensity at high intensity signal regions and become equal to the noise floor at higher frequencies where the signal is completely buried in the noise. The standard deviations in transmittance as well as in the absorption coefficient can be derived from the standard deviations estimated with the model using the intensity spectra of the reference and sample by the error propagation law. The estimated standard deviations fitted well to the standard deviations calculated by the statistical mathematics. This study makes it possible to estimate the accuracies in the transmittance and the optical constants like absorption coefficients obtained from one set of reference and sample data measured with the THz-TDS if we know the constant $C$ in the model which can be derived from a number of the reference data.

\section{Acknowledgement}

The authors would like to express their thanks to Dr. K. Sakai, Dr. S. Saito, Dr. M. Mizuno of NICT, Mr. T. Hirosumi and Dr. H. Ohtake of Aisin Seiki Co. Ltd and Prof. K. Tanaka of Kyoto 
University for their support and advices. This work is supported by the Hamamatsu Optronics Cluster under the Knowledge Cluster Initiative, Ministry of Education, Culture Sports, Science and Technology (MEXT).

\section{References}

[1]. K. Sakai (Ed.), Terahertz Optoelectronics, Springer-Verlag, Berlin Heidelberg, 2005

[2]. S. L. Dexheimer (Ed.), Terahertz Spectroscopy: Principles and Applications, Taylor \& Franchis, New York, 2008

[3]. D. L. Woolard, W. R. Loerop and M. S. Shur (Eds.), Terahertz Sensing Technology, vol. 2: Emerging Scientific Applications and Novel Concepts, World scientific, Singapore, 2003

[4]. D. Grischkowsky, S. Keiding, M. V. Exter and C. Fattinger, J. Opt. Soc. Am. B 7, (1990) 2006-2015.

[5]. M. van Exter and D. Grischkowsky, Appl. Phys. Lett. 56, (1990) 1694-1696.

[6]. A. G. Markelz, A. Roitberg and E. J. Heilweil, Chem. Phys. Lett. 320, (2000) 42-48.

[7]. J. E. Pedersen and S. R. Keiding, IEEE. J. Quantum Electron. 28, (1992) 2518-2522.

[8]. C. J. Strachan, T. Rades, D. A. Newnham, K. C. Gordon, M. Pepper and P. F. Taday, Chem. Phys. Lett. 390, (2004) 20-24.

[9]. W. Withayachumnankul, B. M. Fischer, H. Lin and D. Abbot, J. Opt. Soc. Am. B. 25, (2008) 1059-1072.

[10]. P. U. Jepsen and B. M. Fischer, Opt. Lett. 30, (2005) 29-31.

[11]. M. Naftaly and R. Dudley, Opt. Lett. 34, (2009) 1213-1215

[12]. www.tochigi-nikon.co.jp/technology/terahertz/spectrum/index.htm (in Japanese)

[13]. T. Asahi, T. Yabe and K. Sato, Phys. Stat. Sol. (b). 241, (2004) 648-651.

[14]. S. R. Tripathi, M. Aoki, N. Hiromoto, I. Hosako and T. Asahi, In Proceedings of IEICE General Conference (Kita-Kyushu, Japan, Mar. 2008), 295.

[15]. Z. G. Lu, P. Campbell and X.-C. Zhang, Appl. Phys. Lett. 71, (1997) 593-595.

[16]. P. R. Bevington and D. K. Robinson, Data Reduction and Error Analysis for the Physical Sciences, McGraw-Hill Book, 2003 


\section{Figure captions}

Figure 1.

Frequency-domain reference and sample signals and their standard deviations. Curves $\mathbf{a}$ and $\mathbf{b}$ show the averaged intensity spectra of the reference and sample and curves $\mathbf{c}$ and $\mathbf{d}$ are their respective standard deviations.

Figure 2.

Values of $C$ calculated at various frequencies from the intensities and standard deviations of reference and the $0.767 \mathrm{~mm}$-thick ZnTe sample using Eq. (1).

Figure 3.

Transmittance of $0.767 \mathrm{~mm}$-thick ZnTe sample and its standard deviations. Curve a shows the transmittance, curves $\mathbf{b}$ and $\mathbf{c}$ and are the standard deviations of transmittance computed by the proposed method, statistical mathematics, and curve $\mathbf{d}$ displays the standard deviation from noise floor for comparison. The vertical dashed line shows the detectable frequency limit in the transmittance.

Figure 4.

Absorption coefficients of (a) $0.767 \mathrm{~mm}$ (b) $1.496 \mathrm{~mm}$ (c) $2.976 \mathrm{~mm}$-thick ZnTe samples and their standard deviations computed from the proposed model and statistical mathematics. The vertical dashed line shows the detectable frequency limit in the absorption coefficients. The frequency limit decreases with the sample thickness. 
Figure 1.

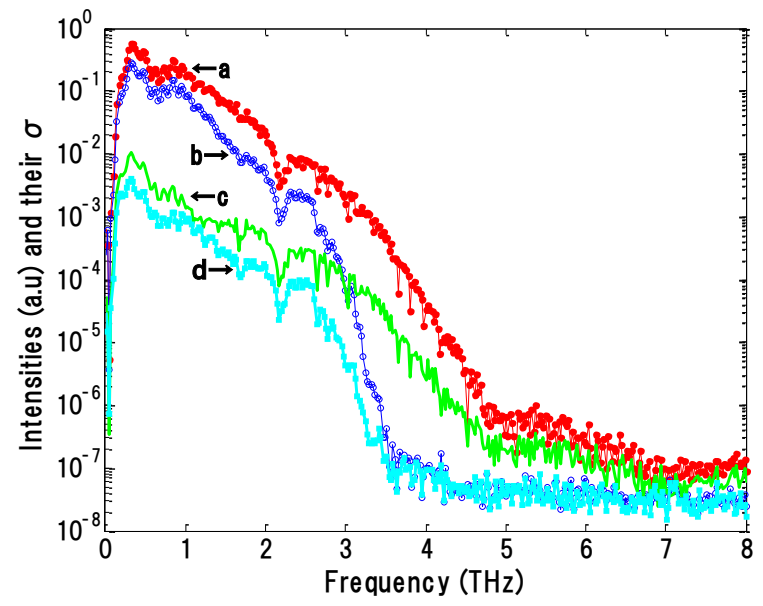


Figure 2

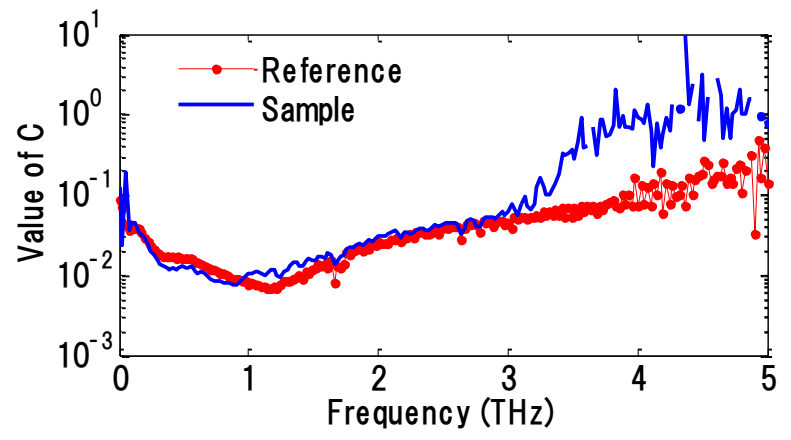


Figure 3

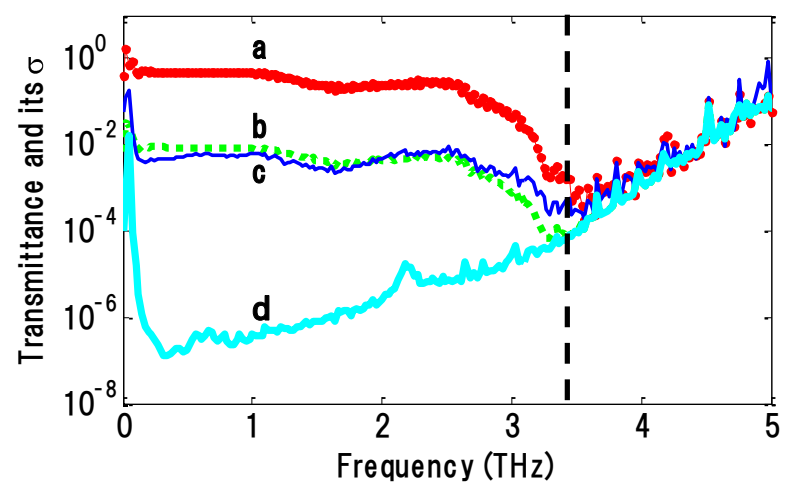


Figure 4 .

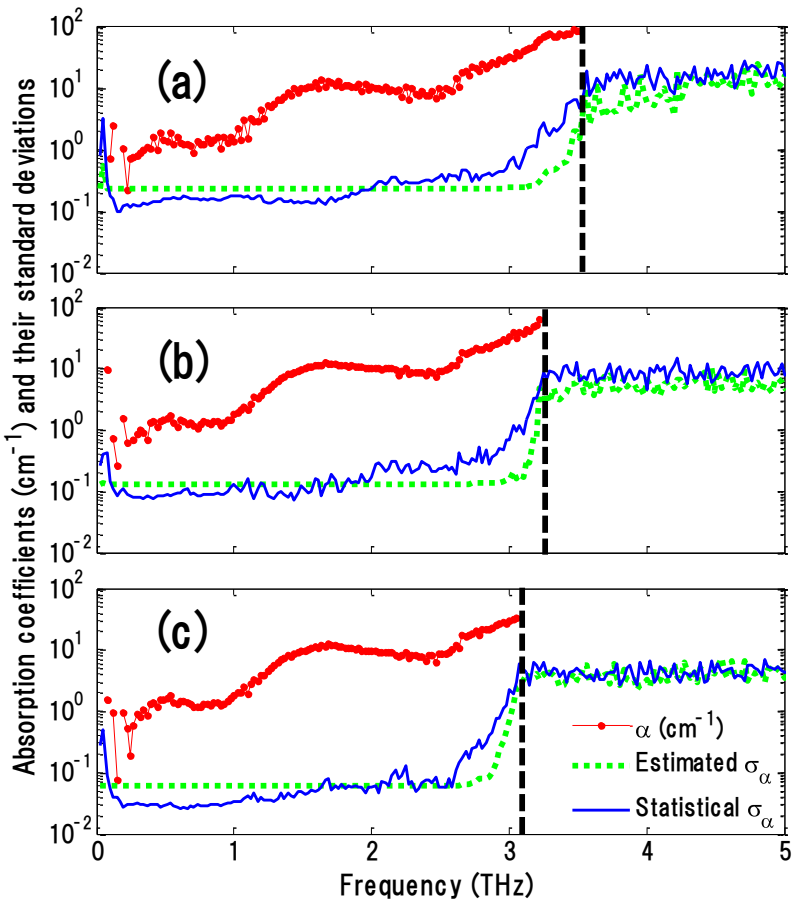

